\title{
Effect of Chronic High-Dose Exogenous Cortisol on Hippocampal Neuronal Number in Aged Nonhuman Primates
}

\author{
James B. Leverenz, ${ }^{1,3,4}$ Charles W. Wilkinson, ${ }^{2,4}$ Molly Wamble, ${ }^{1}$ Shannon Corbin, ${ }^{1}$ Jo Ellen Grabber, ${ }^{5}$ \\ Murray A. Raskind, ${ }^{1,4}$ and Elaine R. Peskind ${ }^{1,4}$ \\ Veterans Affairs Puget Sound Health Care System, ${ }^{1}$ Mental Illness Research, Education, and Clinical Center and ${ }^{2}$ Geriatric \\ Research, Education, and Clinical Center, Seattle, Washington 98108, and Departments of ${ }^{3}$ Neurology and ${ }^{4} P$ sychiatry \\ and Behavioral Sciences, University of Washington School of Medicine, Seattle, Washington 98195, and 5 Washington \\ Regional Primate Research Center, Seattle, Washington 98195
}

Chronic exposure to increased glucocorticoid concentrations appears to lower the threshold for hippocampal neuronal degeneration in the old rat. It has been proposed that increased brain exposure to glucocorticoids may lower the threshold for hippocampal neuronal degeneration in human aging and Alzheimer's disease. Here, we asked whether chronic administration of high-dose cortisol to older nonhuman primates decreases hippocampal neuronal number as assessed by unbiased stereological counting methodology. Sixteen Macaca nemestrina (pigtailed macaques) from 18 to 29 years of age were age-, sex-, and weight-matched into pairs and randomized to receive either high-dose oral hydrocortisone (cortisol) acetate (4-6 mg/kg/d) or placebo in twice daily palatable treats for 12 months. Hypothalamic-pituitary-adrenal activity was monitored by measuring plasma adrenocorticotropin and cortisol, $24 \mathrm{hr}$ urinary cortisol, and CSF cortisol. Urinary, plasma, and CSF cortisol were elevated, and plasma adrenocorticotropin was reduced in the active treatment group. Total hippocampal volume, subfield volumes, subfield neuronal density, and subfield total neuronal number did not differ between the experimental groups. These findings suggest that chronically elevated cortisol concentrations, in the absence of stress, do not produce hippocampal neuronal loss in nonhuman primates.

Key words: cortisol; aging; nonhuman primate; hippocampus; stereology; CSF cortisol
Rodent studies suggest that prolonged exposure to elevated glucocorticoid (GC) concentrations lowers the threshold for hippocampal neuronal degeneration and loss (Sapolsky et al., 1985). A few studies suggest GC neurotoxic effects in primates (Uno et al., 1989; Sapolsky et al., 1990). Older animals may be particularly vulnerable to this phenomenon (Landfield et al., 1981; Kerr et al., 1991). Based on these observations, concern has been raised that prolonged elevation of endogenous GCs caused by chronic stress or pharmacological doses of GCs commonly administered to humans with inflammatory or bronchospastic diseases might produce hippocampal neuronal loss and resultant cognitive impairment, particularly in later life (Keenan et al., 1996; Sapolsky, 1996).

Most in vivo studies addressing GC effects on hippocampal neuronal integrity have used either chronic stress or adrenalectomy versus intact animal paradigms and have attributed effects on the hippocampus to changes in endogenous GC levels produced by these paradigms (Uno et al., 1989; Kerr et al., 1991; Watanabe et al., 1992; Magariños et al., 1997; VollmannHonsdorf et al., 1997). Few studies actually have quantified the effects of chronic exogenous GC administration on hippocampal

Received July 10, 1998; revised Dec. 1, 1998; accepted Jan. 5, 1999.

This work was supported by National Institutes of Health Grant 2P51RR00166, National Institute on Aging Grant AGO6136, the Alzheimer's Association, and the Department of Veterans Affairs. We gratefully acknowledge the assistance of Bradley T. Hyman and T. Gomez-Isla with the stereological methods, the assistance of William R. Morton as Director of the Washington Regional Primate Research Center, and the tireless technical assistance of Lynne Greenup, Elizabeth Colasurdo, Richard Vertz, Judy Johnson, and Mark Murchison.

Correspondence should be addressed to Dr. Elaine R. Peskind, Veterans Affairs Puget Sound Health Care System, Mental Illness Research, Education and Clinical Center (116 MIRECC), 1660 South Columbian Way, Seattle, WA 98108.

Copyright (C) 1999 Society for Neuroscience $\quad 0270-6474 / 99 / 192356-06 \$ 05.00 / 0$ neuronal number in adult animals, and these few studies have produced inconsistent results. Loss of CA3 hippocampal neurons in young rats was reported after 3 months of corticosterone administration that achieved corticosterone concentrations approximating those seen during acute stress (Sapolsky et al., 1985). In contrast, there was no loss or shrinkage of hippocampal CA1 or CA3 neurons in middle-aged rats after 3 months of high-dose exogenous corticosterone administration (Bodnoff et al., 1995). In the only study that evaluated chronic exogenous GC effects in primates (Sapolsky et al., 1990), hippocampal neuronal loss was not observed in young vervet monkeys 1 year after cortisolcontaining pellets had been implanted stereotactically into their hippocampi. None of these exogenous GC administration studies used stereological counting methods to determine neuronal numbers in a manner unbiased by neuron size, shape, or volumetric changes (West and Gundersen, 1990).

Studies in nonhuman primates are more likely to be relevant than rodent studies for evaluating the potential hippocampal neurotoxicity of high-dose GC therapy in older humans or the potential role of chronically elevated endogenous GC in the hippocampal neuronal loss of Alzheimer's disease (Raskind et al., 1982; Peskind et al., 1995). The present study tested the hypothesis that older nonhuman primates receiving chronic highdose cortisol for 1 year would have fewer hippocampal pyramidal neurons than an age-matched control group receiving chronic placebo for 1 year. Neuronal counts were determined by unbiased stereological counting methods (West and Gundersen, 1990).

\section{MATERIALS AND METHODS}

Animals. Sixteen retired breeder pigtailed macaques (Macaca nemestrina $)$ in middle to late life [mean age, $23.1 \pm 1.0$ years $($ mean \pm SEM $)$ 
at termination of experiment; ranged from 18 to 29 years] were selected from the Washington Regional Primate Research Center. There were five males and 11 females. They had been housed in a reproduction colony before the experimental protocol. For the protocol, all were individually housed in the same room. Housing temperature and humidity conditions conformed to the Animal Welfare Act and Guide for the Care and Use of Laboratory Animals. All animals were in good general health. Purina monkey chow was provided during the protocol on a twice daily basis, with fruit supplements. The Washington Regional Primate Research Center is fully accredited by American Association for the Assessment and Accreditation of Laboratory Animal Care International, and all procedures were reviewed and approved by the University of Washington Animal Care and Use Committee.

Treatment protocol. The monkeys were divided into eight pairs matched as closely as possible for age, gender, and weight. Each pair was randomized to receive either high-dose hydrocortisone (cortisol) acetate or placebo for 12 months. The groups did not differ in age (cortisoltreated, $23.1 \pm 1.3$ years at time of death; placebo, $23.1 \pm 1.5$ years at time of death) or pretreatment weight (cortisol-treated, $11.1 \pm 1.7 \mathrm{~kg}$; placebo, $9.8 \pm 1.9 \mathrm{~kg} ; p=0.63$ ). Because there were 11 females and five males, the groups could not be matched evenly for gender (cortisoltreated, five females and three males; placebo, six females and two males). The initial dose of cortisol was $3.85 \mathrm{mg} / \mathrm{kg} / \mathrm{d}$ (hydrocortisone acetate; Upjohn, Kalamazoo, MI). This cortisol dose is approximately equivalent to an adult human receiving the commonly prescribed synthetic GC, prednisone, at a dose of $60 \mathrm{mg} / \mathrm{d}$, a very high therapeutic dose (Schimmer and Parker, 1996). Cortisol was administered by mouth twice per day in a highly palatable treat containing peanut butter, molasses, mashed potato flakes, and ground monkey chow. After 2 weeks of treatment, the cortisol dose was increased to $5.78 \mathrm{mg} / \mathrm{kg} / \mathrm{d}$ in four cortisol treatment condition monkeys in which plasma adrenocorticotropin (ACTH) was inadequately suppressed.

Neuroendocrine measures. Plasma cortisol, plasma ACTH, and $24 \mathrm{hr}$ urinary cortisol were measured during the week before the beginning of drug treatment (baseline), after 2 weeks, and after 3, 6, 9, and 12 months of treatment. After a $24 \mathrm{hr}$ adaptation period, total $24 \mathrm{hr}$ urine for cortisol measurement was collected in a metabolic cage designed for that purpose. Containers placed on solid $\mathrm{CO}_{2}$ were positioned under a collection spout and replaced at frequent intervals. Urine containers remained frozen until thawed for volume determinations. All containers from a given monkey for the $24 \mathrm{hr}$ sampling interval were mixed thoroughly after thawing. After volume measurement, $3 \mathrm{ml}$ aliquots of urine for each monkey from each sampling period were stored frozen at $-70^{\circ} \mathrm{C}$. Urine samples $(500 \mu \mathrm{l})$ were extracted by mixing thoroughly with $1 \mathrm{ml}$ of chilled methylene chloride, removing an aliquot of the organic phase, and drying. Dried samples were reconstituted with buffer and assayed as described previously for plasma cortisol (Wilkinson et al., 1997). Creatinine was determined by a quantitative colorimetric method (Stanbio Laboratory, San Antonio, TX).

Blood samples for plasma cortisol and ACTH measurements were collected from sedated monkeys (ketamine hydrochloride, $10 \mathrm{mg} / \mathrm{kg}$ ) using a "squeeze cage" technique by which monkeys were briefly immobilized to allow venipuncture. Blood samples for cortisol and ACTH measurements were drawn within $10 \mathrm{~min}$ of sedation of animals. All sampling was done between 9:00 and 10:15 A.M. Blood was stored on ice in chilled polystyrene tubes and cold centrifuged within $1 \mathrm{hr}$ of sample collection; plasma was then separated and stored at $-70^{\circ} \mathrm{C}$ until assayed. Plasma cortisol and plasma ACTH were measured by radioimmunoassay as described previously (Wilkinson et al., 1997).

Monkeys were killed during an $8 \mathrm{hr}$ period on 3 consecutive days, with animals from cortisol and placebo groups alternating in sequence. CSF was obtained after prekilling sedation by either lumbar or cisternal puncture. Cortisol concentration was measured in $100 \mu \mathrm{l}$ aliquots of unextracted CSF with a ${ }^{125}$ I-radioimmunoassay kit (Pantex, Santa Monica, CA). All samples were measured in the same assay. Interassay and intra-assay coefficients of variation for this method in our laboratory are 7.1 and $3.7 \%$, respectively. To evaluate possible effects of elapsed time from A.M. cortisol dose on cortisol concentrations achieved in the central compartment, we performed Pearson product-moment correlations within treatment groups between $\Delta$ time from A.M. cortisol dose to CSF sampling. Correlations were nonsignificant $(r=0.199$ and 0.273 for cortisol and placebo groups, respectively). Therefore, there is no indication that a marked fluctuation in CSF cortisol levels occurred during the $12 \mathrm{hr}$ between successive cortisol doses during the course of the study.
Tissue preparation. After 12 months of treatment, the animals were killed by valium-phenobarbitol injection. Brains were then rapidly removed and sectioned into $0.5-\mathrm{cm}$-thick coronal blocks of the cerebral hemispheres and $0.5-\mathrm{cm}$-thick horizontal blocks of the brainstem. The right hemispheric blocks were fixed flat (to maintain gross morphology) between $4 \%$ paraformaldehyde-soaked sponges for $36 \mathrm{hr}$ and then stored in PBS, pH 7.4, with $20 \%$ sucrose and $0.02 \%$ sodium azide. Fixed blocks of temporal lobe from the right hemisphere were serially sectioned on a cryostat at $50 \mu \mathrm{m}$. Every twelfth section (600 $\mu \mathrm{m}$ interval) was taken for thionin staining. The left hemisphere and brainstem were rapidly frozen between cooled aluminum plates in $\mathrm{a}-70^{\circ} \mathrm{C}$ freezer and stored at that temperature for future studies.

Stereological counting techniques. All counting was performed blind to treatment condition. Principles of stereology were used to select and count neurons within the subfields of the hippocampus (West and Gundersen., 1990; West, 1993a). After randomly selecting a starting point rostral to the hippocampus, sections were taken at $600 \mu \mathrm{m}$ intervals and thionin stained for cell counts. The hippocampal subfield boundaries were outlined (Fig. 1) on each stained slide for all cases according to previously published criteria (Rosene and Van Hoesen, 1987). These fields included the dentate granule cell layer, dentate hilus, CA2/3, CA1 (including prosubiculum), and subiculum. Neuronal density within the individual sections was based on counts using the optical disector technique with a Nikon (Tokyo, Japan) Optiphot-2 microscope with a chromatic aberration-free, $\mathrm{N}$-series (CF N) Plan Apochromat $100 \times(1.4 \mathrm{NA})$ oil objective, a digital linear measuring system for $x$ - and $y$-axes (Boeckeler, Tucson, AZ), and a video camera (DAGE-MTI, Michigan City, IN) output to a high-resolution video screen (Sony, Tokyo, Japan). An unbiased counting frame with extended exclusion lines $(25 \times 25 \mu \mathrm{m}$ for all fields, except the dentate granule cell layer for which $10 \times 10 \mu \mathrm{m}$ was used) was printed on an acetate sheet (calibrated with a slide micrometer) and placed over the video screen. For each section counted, a grid of potential disectors was laid out over the entire section. A random starting point was used for the first disector selected, and then subsequent disectors were systematically sampled from that point. The frequency of disector sampling was dependent on the neuronal density for each hippocampal subfield. For example, the number of disectors chosen in a section for the dentate granule cells was greater than for CA1 because the likelihood of a particular disector including the smaller granule cell layer (by area) was much less. This method allowed for random but systematic disector sampling for each subfield. In all subfields, at least 100 disectors were counted. Subfield volumes were calculated by determining the subfield areas on all sections counted, using a computerized image analysis system (MCID; Imaging Research Inc., Ontario, Canada), and multiplying by $600 \mu \mathrm{m}$. An unbiased estimate of total number of neurons within each subfield was derived from the product of the neuronal density and the estimated field volume $\left(\mathrm{N}=\mathrm{N}_{\mathrm{v}} \times \mathrm{V}_{\text {ref }}\right)$.

Neurohistology. Light microscopic examination for neuropathology was performed (by J. B. Leverenz) on all thionin-stained slides. Slides were examined blind to treatment condition.

Statistical analyses. Values are expressed as mean \pm SEM. Differences in neuroendocrine measures over time within each treatment group were evaluated by repeated measures ANOVA. A significant difference over time prompted paired $t$ test comparisons between each treatment time point value and the baseline value in that group. Differences in neuroendocrine measures between treatment groups at each time point were evaluated by unpaired $t$ tests. Differences in hippocampal subfield neuronal counts and volumes between groups also were evaluated by unpaired $t$ tests. To minimize the likelihood of type II error, no correction was made for multiple comparisons.

\section{RESULTS}

\section{Animals' general condition during study}

All monkeys survived the 12 month treatment period in good general health, except for one of the cortisol-treated monkeys. This monkey died of Klebsiella sepsis 2 weeks after the 9 month evaluation point. Although this monkey's brain was removed within 60 min of death, the brain was not used for the analysis of neuronal number in the hippocampal formation. The 12 month weight and weight gain of the placebo-treated monkeys $(9.8 \pm 1.9$ and $0.4 \pm 0.3 \mathrm{~kg}$ ) did not differ from the 12 month weight and weight gain of the cortisol-treated monkeys $(11.1 \pm 1.7$ and $1.0 \pm$ 
A

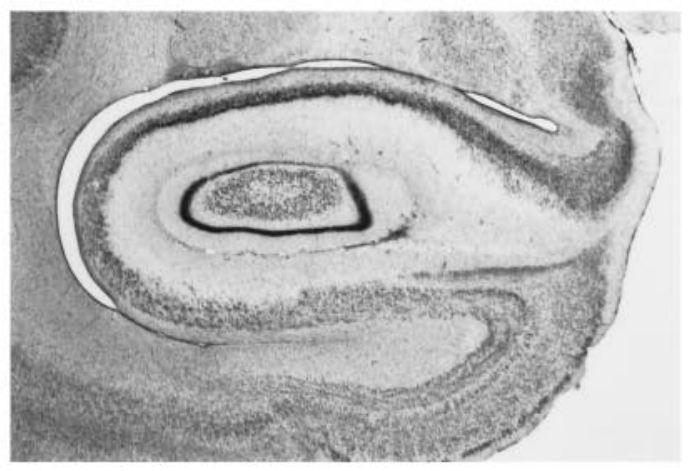

C

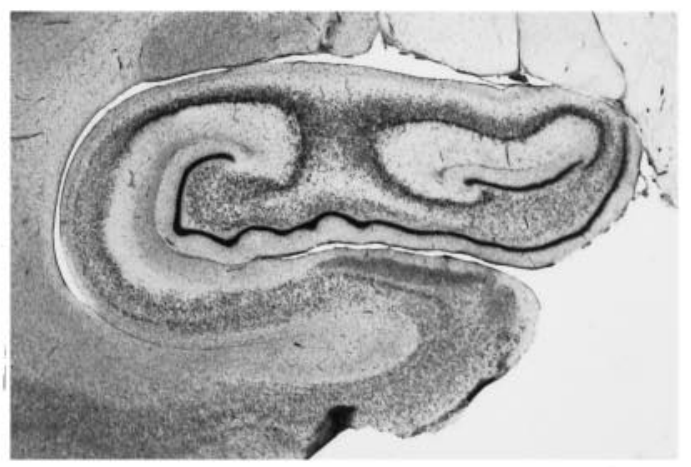

$\mathrm{E}$

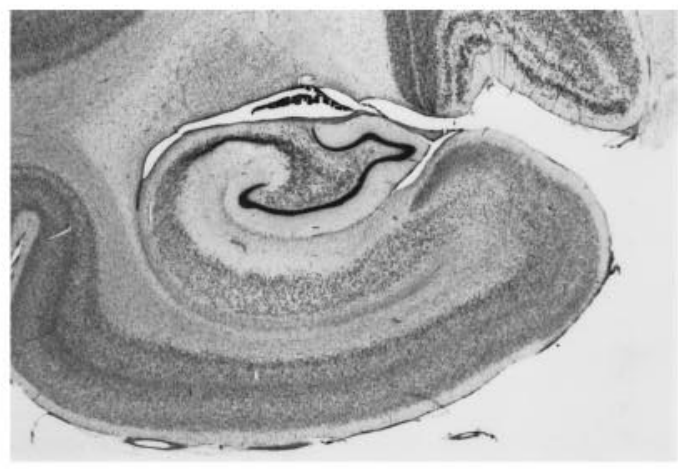

G

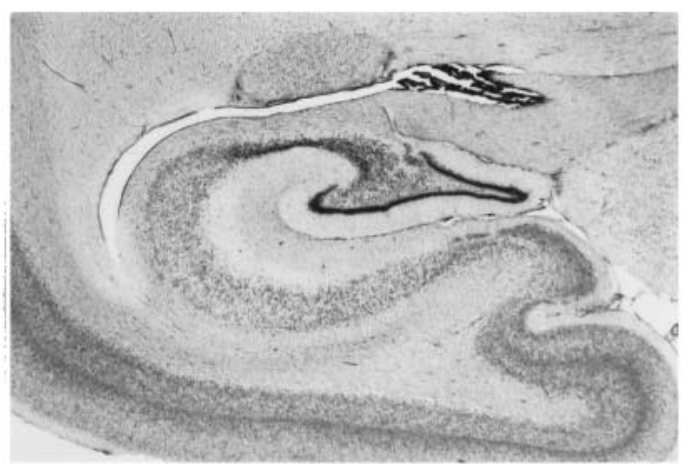

B

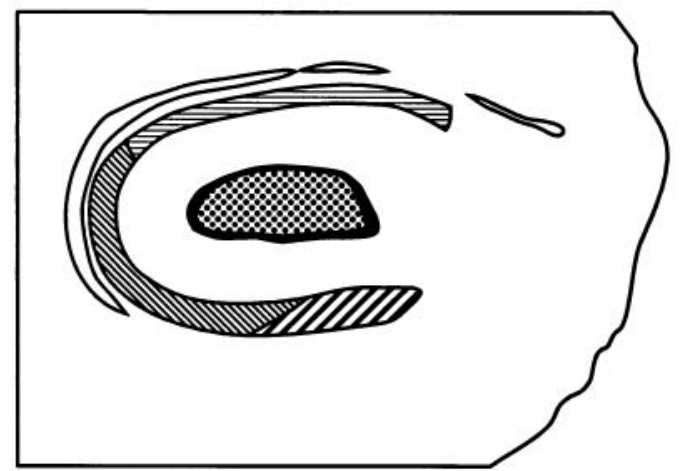

D

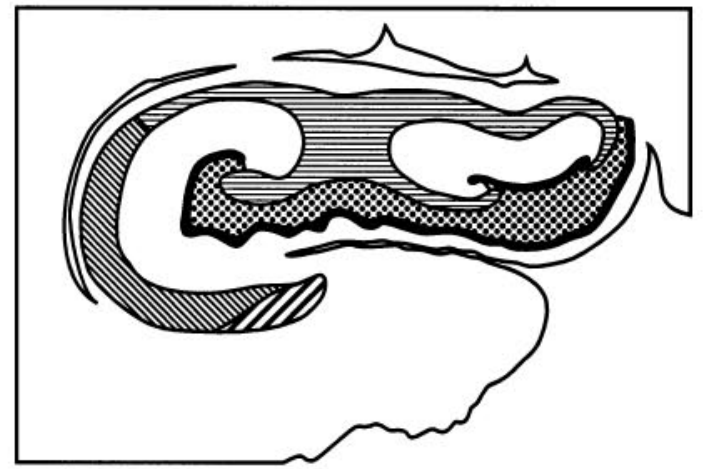

F

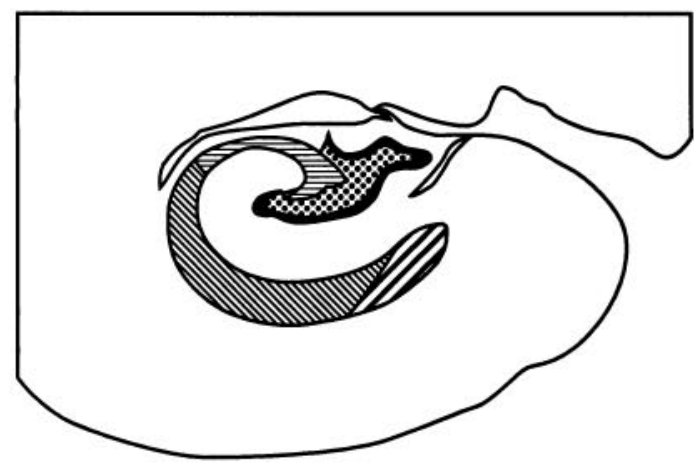

$\mathrm{H}$

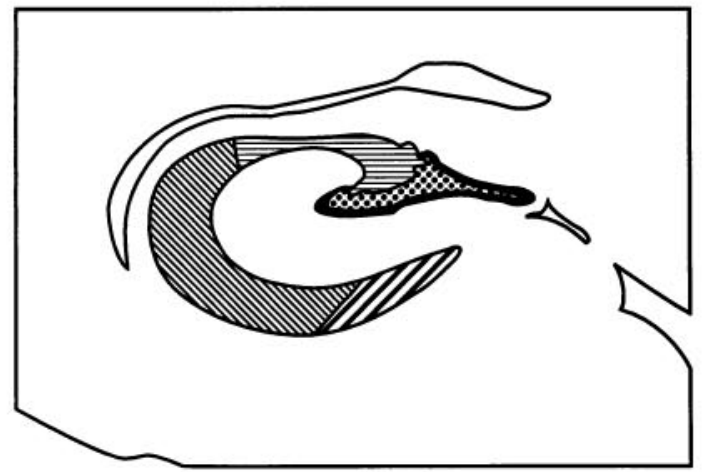

Figure 1. Representative coronal sections from rostral to caudal hippocampus $(A, C, E, G)$ and outlines of dentate granule cell layer, hilus, CA2/3, CA1, and subiculum as used in stereological cell counting $(B, D, F, H)$. CA1' (Rosene and Van Hoesen, 1987) was not included within the assessed CA1 subfield. 


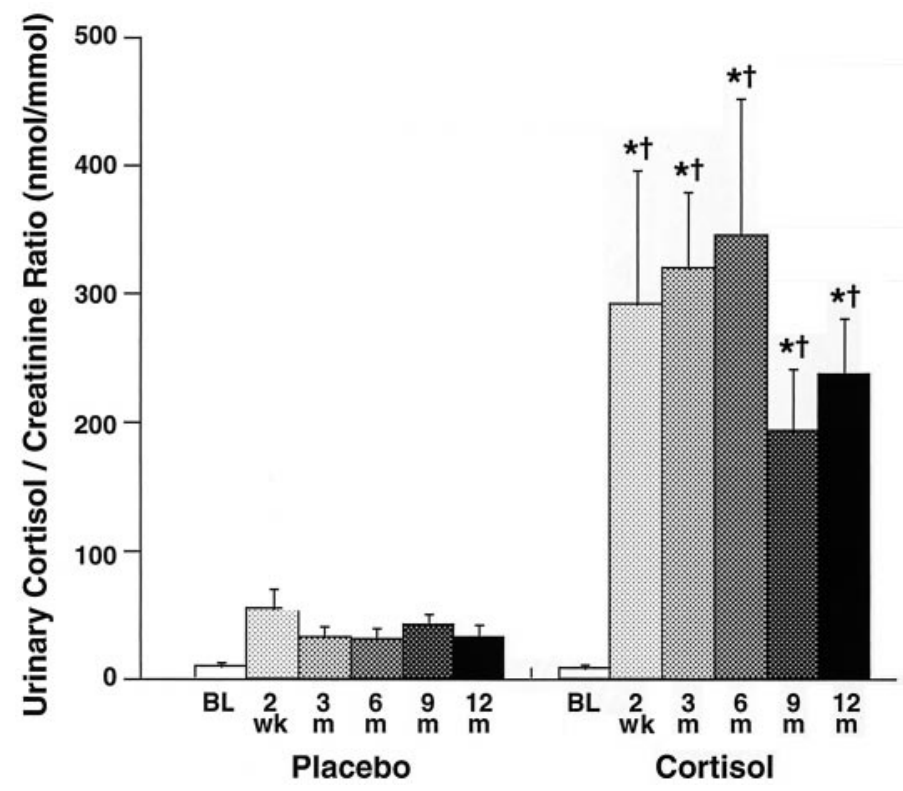

Figure 2. Twenty-four hour urinary cortisol concentrations (expressed as nanomoles per millimoles creatinine) in placebo- and cortisol-treated groups at baseline $(B L)$, after 2 weeks $(w k)$, and after 3, 6, 9, and 12 months $(m)$ of treatment. ${ }^{*} p<0.05$, higher than baseline; $\uparrow p<0.01$, higher than placebo group.

$0.4 \mathrm{~kg})(t=0.87 ; p=0.40$; and $t=0.82 ; p=0.43$, respectively, for end weights and weight gain). Brain weights did not differ between cortisol-treated monkeys $(93.0 \pm 2.6 \mathrm{gm})$ and placebotreated monkeys $(99.5 \pm 4.0 \mathrm{gm})(t=1.32 ; p=0.21)$.

\section{Endocrine effects of cortisol treatment}

Cortisol-treated monkeys tended toward greater, but not statistically significant, weight gain (see previous section) versus the placebo group. Many of the cortisol-treated monkeys, but not those in the placebo group, developed cushingoid features (facial puffiness, buffalo hump). Two of the cortisol-treated animals developed mild glucose intolerance, but none required insulin treatment.

Twenty-four hour urinary cortisol excretion (expressed as nanomoles of cortisol per millimoles of creatinine) at baseline, after 2 weeks, and after 3, 6, 9, and 12 months of treatment are presented in Figure 2. Baseline pretreatment 24 hr urinary cortisol did not differ between cortisol and placebo groups. Within the cortisol-treated group, $24 \mathrm{hr}$ urinary cortisol was significantly increased from baseline at all time points $(p<0.05)$. Twentyfour hour urinary cortisol was significantly higher in the cortisoltreated group compared with the placebo group at 2 weeks and 3 , 6 , 9, and 12 months of treatment $(p<0.01)$. Plasma cortisol concentrations in the cortisol treatment group were significantly higher than baseline values during exogenous cortisol administration at all time points $(p<0.01)$ and significantly higher than in the placebo treatment group at 3, 6, 9, and 12 months of treatment $(p<0.05)$ (data not shown).

Plasma ACTH concentrations at the same time points are presented in Figure 3. Plasma ACTH did not differ between groups at baseline $(p=0.12)$. Exogenous cortisol administration appropriately suppressed plasma ACTH concentrations, which were significantly lower than baseline at all treatment time points in the cortisol-treated group $(p<0.01)$. Plasma ACTH in the cortisol-treated group was significantly lower than in the placebo

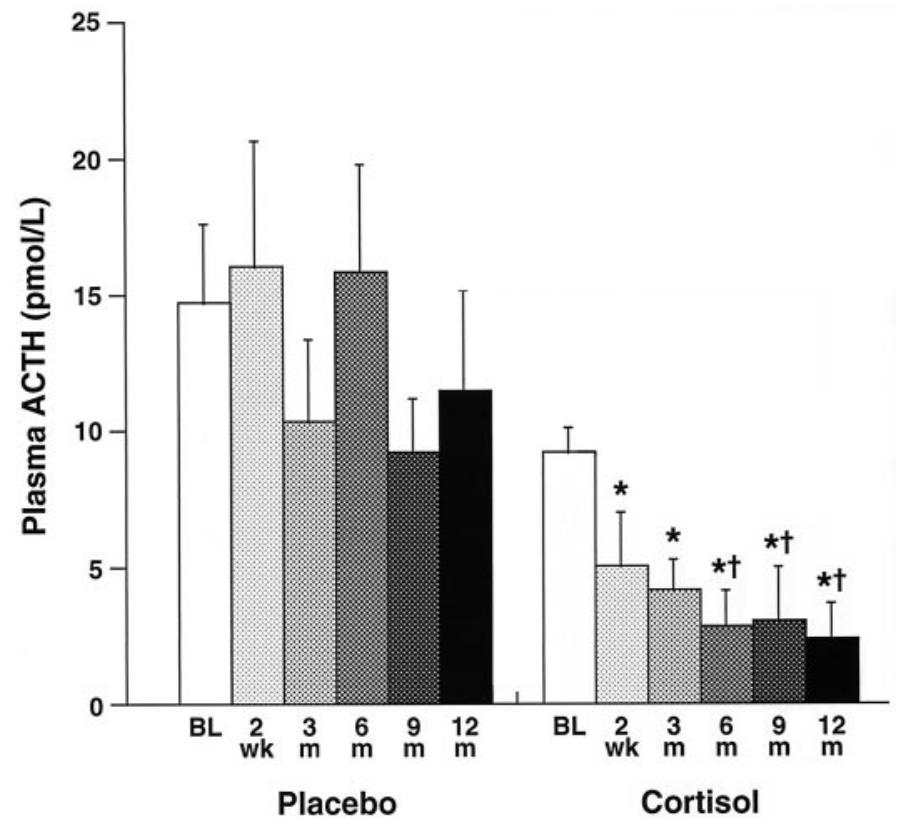

Figure 3. Plasma ACTH concentrations (expressed as picomoles per liter) in placebo- and cortisol-treated groups at baseline $(B L)$, after 2 weeks $(w k)$, and after $3,6,9$, and 12 months $(m)$ of treatment. ${ }^{*} p<0.01$, lower than baseline; $t p<0.05$, lower than placebo group.

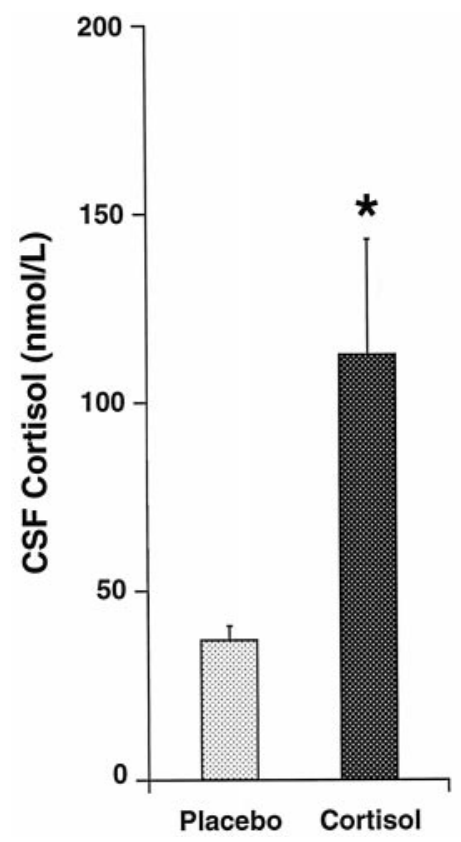

Figure 4. CSF cortisol concentrations (expressed as nanomoles per liter) in placebo- and cortisol-treated groups after 12 months of treatment obtained by lumbar or cisternal puncture in sedated animals before death. $* p<0.01$, higher in placebo group.

group at 6, 9, and 12 months of treatment $(p<0.05)$. Plasma ACTH did not differ over time in the placebo group.

CSF cortisol concentrations before death are presented in Figure 4. CSF cortisol levels were significantly higher in the cortisol-treated group than in the placebo group $(p<0.01)$.

Hippocampal subfield neuronal numbers and volumes Hippocampal subfield neuronal numbers, volumes, and density are presented in Table 1 . There were no significant differences in 


\begin{tabular}{|c|c|c|c|}
\hline & Placebo & Cortisol & $p$ value* \\
\hline \multicolumn{4}{|l|}{ Subiculum } \\
\hline Volume & $29.4 \pm 2.6$ & $25.5 \pm 1.4$ & 0.12 \\
\hline Density & $7165.9 \pm 377.4$ & $7851.1 \pm 504.7$ & 0.86 \\
\hline Cell \# & $208,299 \pm 15,346$ & $195,969 \pm 9772$ & 0.26 \\
\hline \multicolumn{4}{|l|}{ CA1 } \\
\hline Volume & $67.7 \pm 4.1$ & $71.7 \pm 2.7$ & 0.78 \\
\hline Density & $10,281.6 \pm 345.3$ & $10,183.9 \pm 332.5$ & 0.42 \\
\hline Cell \# & $694,803 \pm 46,471$ & $726,092 \pm 16,805$ & 0.72 \\
\hline \multicolumn{4}{|l|}{$\mathrm{CA} 2 / 3$} \\
\hline Volume & $37.9 \pm 2.2$ & $40.6 \pm 2.7$ & 0.78 \\
\hline Density & $8736.3 \pm 374.2$ & $8797.4 \pm 276.9$ & 0.55 \\
\hline Cell \# & $329,104 \pm 18,289$ & $358,868 \pm 29,680$ & 0.80 \\
\hline \multicolumn{4}{|l|}{ Hilus } \\
\hline Volume & $49.5 \pm 4.1$ & $52.1 \pm 3.2$ & 0.68 \\
\hline Density & $5331.6 \pm 127.0$ & $5060.1 \pm 163.6$ & 0.10 \\
\hline Cell \# & $262,668 \pm 20,166$ & $264,355 \pm 20,534$ & 0.52 \\
\hline \multicolumn{4}{|l|}{ Dentate } \\
\hline Volume & $13.2 \pm 0.8$ & $14.4 \pm 1.0$ & 0.81 \\
\hline Density & $167,937.6 \pm 8841.6$ & $172,272 \pm 5777.4$ & 0.65 \\
\hline Cell \# & $2,216,385 \pm 173,760$ & $2,460,120 \pm 141,029$ & 0.84 \\
\hline
\end{tabular}

*Student's $t$ test, unpaired, one-tailed.

neuronal number between groups in any hippocampal subfield $(p>0.20)$ nor were there significant differences in subfield volumes or densities between treatment groups $(p>0.10)$. Total hippocampal volumes between groups did not significantly differ $(p=0.64)$ (data not shown).

\section{Neurohistology}

There were no apparent differences in perikaryal size, nuclear size, or nuclear density between treatment groups in any hippocampal subfield. The regularity of pyramidal cell layers was indistinguishable between groups.

\section{DISCUSSION}

These results do not confirm the hypothesis that high-dose chronic exogenous GC administration produces loss of hippocampal neurons in older nonhuman primates. The threefold increase in CSF cortisol found in the treated monkeys indicates that substantial elevations in cortisol concentration were achieved in the CNS. There were no effects of cortisol treatment on neuronal number, density, or volume of any hippocampal subfield. In particular, there was no suggestion that cortisol treatment reduced neuronal number in $\mathrm{CA} 2 / 3$, the area considered most vulnerable to GC neurotoxic effects (Landfield et al., 1981; Sapolsky et al., 1985). The subiculum also did not show evidence of significant neuronal loss, despite its apparent susceptibility to neuronal loss with normal aging (West, 1993b; Simic et al., 1997).

The current study is not alone in failing to demonstrate that chronic GC administration produces hippocampal neuronal loss. Bodnoff et al. (1995) administered exogenous corticosterone to middle-aged rats for 3 months at doses that mimicked corticosterone concentrations normally achieved at the diurnal peak or the even higher concentrations normally achieved during stress. Although corticosterone-treated rats demonstrated spatial learning impairment and electrophysiological changes, suggesting impaired hippocampal synaptic plasticity, there were no effects of corticosterone treatment on hippocampal neuronal number, neuronal size, or subfield volume, nor were any other neurohistological abnormalities noted. Sapolsky et al. (1990) implanted a cortisol-containing pellet in one hippocampus and a cholesterolcontaining placebo pellet in the other hippocampus of four young vervet monkeys. After 1 year, there were no differences in hippocampal subfield neuronal number between the cortisol pelletexposed side and the cholesterol pellet-exposed side. Qualitative changes interpreted as consistent with neurodegeneration, including shrinkage and condensation of soma and nucleus, dendritic atrophy, and cell layer irregularity, were more intense and frequent in the cortisol pellet-exposed hippocampal CA2/3 subfields. Neither systemic nor local hippocampal cortisol concentrations were determined in this study. Neither of these studies confirmed Sapolsky et al.'s (1985) report in rats of hippocampal neuronal loss after chronic exogenous GC administration, but they suggest other effects of exogenous GC on hippocampal neuronal integrity. Consistent with this possibility is the demonstration in single-section Golgi preparations that exogenous corticosterone administration to rats produces atrophy of the apical dendritic tree of CA3 pyramidal neurons (Woolley et al., 1990; Watanabe et al., 1992). Although no evidence of neuronal morphological abnormalities with exogenous cortisol treatment was found in the present study, further studies of dendritic morphology may reveal cortisol effects.

Several studies have inferred GC-induced hippocampal neuronal loss in chronically stressed older rats (Kerr et al., 1991), chronically stressed orchiectomized rats (Mizoguchi et al., 1992), and socially subordinate wild vervet monkeys that died spontaneously during episodes of apparent severe stress (Uno et al., 1989). In the latter naturalistic monkey study, cortisol concentrations were not determined, and decreased neuronal numbers were found only in CA3 in the subordinate male vervets. That these reported effects of stress on hippocampal neuronal number can be attributed to the elevated GC levels accompanying stress is a viable hypothesis (Sapolsky, 1992), but other effects of stress could also have accounted for these effects on hippocampal neuronal integrity. None of the above stress paradigm studies used unbiased stereological counting techniques. A recent study that used unbiased stereological counting techniques to determine the effects of a $28 \mathrm{~d}$ "psychological conflict" stress paradigm on hippocampal neuronal number in the tree shrew, a phylogenetic intermediate between insectivores and primates (Martin, 1990), did not confirm stress-induced hippocampal neuronal loss (Vollmann-Honsdorf et al., 1997). This stress paradigm increased urinary cortisol concentrations more than threefold, but there was no effect on neuronal numbers in the hippocampal CA3 or CA1 subfields. This psychosocial conflict paradigm in tree shrews has produced CA3 pyramidal neuron apical dendritic atrophy, as determined by the single-section Golgi technique (Magariños et al., 1997). Whether the CA3 apical dendritic atrophy and alterations in mossy fiber CA3 synaptic ultrastructure after chronic stress represent early signs of neuronal degeneration or adaptive and reversible responses to stress and/or increased GC concentrations remains unclear (Magariños et al., 1997).

Application of unbiased stereological counting techniques to nonhuman primate studies has failed to demonstrate hippocampal neuronal loss after exogenous cortisol administration in macaques in the current study or after chronic stress associated with increased cortisol secretion in tree shrews (Vollmann-Honsdorf et al., 1997). These results do not rule out the possibility that GC-induced modifications of hippocampal neuronal structure or 
function contribute to the cognitive deficits reported in persons receiving acute and chronic GC therapy and in persons with Cushing's disease or that hypothalamic-pituitary-adrenal axis hyperactivity may contribute to exacerbation of cognitive and noncognitive abnormalities in aging and Alzheimer's disease (Starkman et al., 1992; Newcomer et al., 1994; Raskind et al., 1994; Keenan et al., 1996; Wolkowitz et al., 1997). However, they provide some reassurance that therapeutic use of GCs in older humans is unlikely to produce hippocampal neuronal death as a common adverse effect. Further studies in nonhuman primates and humans will be necessary to evaluate the possible effects of stress-associated endogenous cortisol elevations and agingassociated changes in hypothalamic-pituitary-adrenal axis regulation on hippocampal structure and function in human aging and Alzheimer's disease.

\section{REFERENCES}

Bodnoff SR, Humphreys AG, Lehman JC, Diamond DM, Rose GM, Meaney MJ (1995) Enduring effects of chronic corticosterone treatment on spatial learning, synaptic plasticity, and hippocampal neuropathology in young and middle-aged rats. J Neurosci 15:61-69.

Keenan PA, Jacobson MW, Soleymani RM, Mayes MD, Stress ME, Yaldoo DT (1996) The effect on memory of chronic prednisone treatment in patients with systemic disease. Neurology 47:1396-1402.

Kerr DS, Campbell LW, Applegate MD, Brodish A, Landfield PW (1991) Chronic stress-induced acceleration of electrophysiologic and morphometric biomarkers of hippocampal aging. J Neurosci 11:1316-1324.

Landfield PW, Baskin RK, Pitler TA (1981) Brain aging correlates: retardation by hormonal-pharmacological treatments. Science 214:581-584.

Magariños AM, Verdugo JM, McEwen BS (1997) Chronic stress alters synaptic terminal structure in hippocampus. Proc Natl Acad Sci USA 94:14002-14008.

Martin RD (1990) Primate origins and evolution, pp 190-213. London: Chapman and Hall.

Mizoguchi K, Kunishita T, Chui D-H, Tabira T (1992) Stress induces neuronal death in the hippocampus of castrated rats. Neurosci Lett 138:157-160.

Newcomer JW, Craft S, Hershey T, Askins K, Bardgett ME (1994) Glucocorticoid-induced impairment in declarative memory performance in adult humans. J Neurosci 14:2047-2053.

Peskind ER, Raskind MA, Wingerson D, Pascualy M, Thal LJ, Dobie DJ, Veith RC, Dorsa DM, Murray S, Sikkema C, Galt SA, Wilkinson CW (1995) Enhanced hypothalamic pituitary adrenocortical axis responses to physostigmine in normal aging. J Gerontol A Biol Sci Med Sci 50:M114-M120.

Raskind MA, Peskind E, Rivard M-F, Veith R, Barnes R (1982) The dexamethasone suppression test and cortisol circadian rhythm in primary degenerative dementia. Am J Psychiatr 139:1468-1471.
Raskind MA, Peskind ER, Wilkinson CW (1994) Hypothalamic-pituitary-adrenal axis regulation and human aging. Ann NY Acad Sci 746:327-335.

Rosene DL, Van Hoesen GW (1987) The hippocampal formation of the primate brain: a review of some comparative aspects of cytoarchitecture and connections. In: Cerebral cortex (Jones EJ, Peters A, eds), pp 345-456. New York: Plenum.

Sapolsky RM (1992) Stress, the aging brain, and the mechanisms of neuron death, pp 119-143. Cambridge, MA: MIT.

Sapolsky RM (1996) Why stress is bad for your brain. Science 273:749-750.

Sapolsky RM, Krey LC, McEwen BS (1985) Prolonged glucocorticoid exposure reduces hippocampal neuron number: implications for aging. J Neurosci 5:1222-1227.

Sapolsky RM, Uno H, Rebert CS, Finch CE (1990) Hippocampal damage associated with prolonged glucocorticoid exposure in primates. J Neurosci 10:2897-2902.

Schimmer BP, Parker KL (1996) Adrenocorticotropic hormone, adrenocortical steroids and their synthetic analogs: inhibitors of the synthesis and actions of adrenocortical hormones. In: Goodman \& Gilman's the pharmacological basis of therapeutics, Ed 9 (Hardman JG, Limbird LE, eds) pp 1459-1485. New York: McGraw-Hill.

Simic G, Kostovic I, Winblad B, Bogdanovic N (1997) Volume and number of neurons of the human hippocampal formation in normal aging and Alzheimer's disease. J Comp Neurol 379:482-494.

Starkman MN, Gebarski SS, Berent S, Schteingart DE (1992) Hippocampal formation volume, memory dysfunction, and cortisol levels in patients with Cushing's syndrome. Biol Psychiatry 32:756-765.

Uno H, Tarara R, Else JG, Suleman MA, Sapolsky RM (1989) Hippocampal damage associated with prolonged and fatal stress in primates. J Neurosci 9:1705-1711.

Vollmann-Honsdorf GK, Flugge G, Fuchs E (1997) Chronic psychosocial stress does not affect the number of pyramidal neurons in tree shrew hippocampus. Neurosci Lett 233:121-124.

Watanabe Y, Gould E, McEwen BS (1992) Stress induces atrophy of apical dendrites of hippocampal CA3 pyramidal neurons. Brain Res 588:341-345.

West MJ (1993a) New stereological methods for counting neurons. Neurobiol Aging 14:275-285.

West MJ (1993b) Regionally specific loss of neurons in the aging human hippocampus. Neurobiol Aging 14:287-293.

West MJ, Gundersen HJG (1990) Unbiased stereological estimation of the number of neurons in the human hippocampus. J Comp Neurol 296:1-22.

Wilkinson CW, Peskind ER, Raskind MA (1997) Decreased hypothalamic-pituitary-adrenal axis sensitivity to cortisol feedback inhibition in human aging. Neuroendocrinology 65:79-90.

Wolkowitz OM, Reus VI, Canick J, Levin B, Lupien S (1997) Glucocorticoid medication, memory and steroid psychosis in medical illness. Ann NY Acad Sci 823:81-96.

Woolley CS, Gould E, McEwen BS (1990) Exposure to excess glucocorticoids alters dendritic morphology of adult hippocampal pyramidal neurons. Brain Res 531:225-231. 